\title{
Estrés, respuestas emocionales, factores de riesgo, psicopatología y manejo del personal de salud durante la pandemia por COVID-19
}

\section{Stress, emotional responses, risk factors, psychopathology and management of healthcare workers during (COVID-19) pandemic}

Sergio Ignacio Muñoz-Fernández, ${ }^{1}$ Diana Molina-Valdespino, ${ }^{2}$ Rosalba Ochoa-Palacios, ${ }^{2}$ Oscar SánchezGuerrero, ${ }^{2}$ Juan Antonio Esquivel-Acevedo ${ }^{2}$

Resumen

ANTECEDENTES: El nuevo coronavirus SARS-Cov-2, causante de la pandemia por COVID-19, afecta al personal de salud por la repercusión emocional que implica enfrentarse a situaciones complejas y estresantes que retan los mecanismos de regulación emocional y capacidad de adaptación a la vida cotidiana para mantener un estado de salud mental óptimo.

OBJETIVO: Describir las reacciones emocionales esperadas ante COVID-19, los factores individuales protectores y de riesgo, la patología psiquiátrica y las distintas intervenciones que pueden efectuarse en apoyo de las diversas reacciones.

CONCLUSIONES: Para los trabajadores de la salud, la pandemia por COVID-19 es un factor de riesgo de problemas de salud mental que requiere evaluación y atención oportuna.

PALABRAS CLAVE: SARS Cov2; Coronavirus; pandemia de COVID-19; trabajadores de la salud; situaciones estresantes; regulación emocional; factores de riesgo.

Abstract

BACKGROUND: The new SARS-Cov-2 coronavirus, causing COVID-19 pandemic, affects healthcare workers, who suffer a high emotional impact, facing complex and stressful situations, challenging their emotional regulation mechanisms and their ability to cope with daily life stress, to keep an optimal state of mental health.

OBIECTIVE: To describe the expected emotional reactions to COVID-19, the protective and risk factors, the psychiatric pathology related to the pandemic and the different treatment interventions that can be carried out to support the possible emotional reactions that can emerge during the crisis.

CONCLUSION: COVID-19 pandemic is a risk for mental disturbances of healthcare workers that must be evaluated and treated as soon as possible.

KEYWORDS: SARS Cov2; Coronavirus; COVID-19 Pandemic; Health Workers; Stressful situations; Emotional Regulation; Risk Factors.

\author{
Jefe del servicio de Salud Mental. \\ ${ }^{2}$ Adscrito al servicio de Salud Mental. \\ Instituto Nacional de Pediatría, Ciudad \\ de México. \\ Recibido: 13 de mayo 2020 \\ Aceptado: 22 de mayo 2020 \\ Correspondencia \\ Sergio Ignacio Muñoz-Fernández \\ sergiomf535@yahoo.com.mx \\ Este artículo debe citarse como \\ Muñoz-Fernández SI, Molina-Valdespi- \\ no D, Ochoa-Palacios R, Sánchez-Gue- \\ rrero O, Esquivel-Acevedo JA. Estrés, \\ respuestas emocionales, factores de \\ riesgo, psicopatología y manejo del \\ personal de salud durante la pandemia \\ por COVID-19. Acta Pediatr Méx 2020; \\ 41 (Supl 1):S127-S136.
}

\section{INTRODUCCIÓN}

La salud mental abarca una amplia gama de actividades directa o indirectamente relacionadas con el componente de bienestar mental, incluido en la definición de salud que da la OMS: "un estado de completo bienestar físico, mental y social, y no solo la ausencia de afecciones o enfermedades".

El nuevo coronavirus SARS-Cov-2, causante de la pandemia por COVID-19, que afecta al mundo, ha alcanzado a México y el personal de salud, comprometido de forma total en el en- 
frentamiento de este problema de salud pública, sufre un alto impacto emocional, al enfrentarse a situaciones complejas y estresantes, que retan los mecanismos de regulación emocional y capacidad de adaptación a la vida cotidiana del personal de salud para mantener un estado de salud mental óptimo. Por ello, tanto a nivel internacional como nacional, se han desarrollado estrategias para brindar apoyo para la salud mental del personal sanitario, aplicando pruebas de tamizaje para detectar manifestaciones de psicopatología que pudieran poner en riesgo a los trabajadores de la salud y algoritmos de manejo para las mismas.

El Instituto Nacional de Pediatría cuenta con un área de triage y un área COVID para casos pediátricos. El objetivo de este artículo es brindar al personal de salud las herramientas para la comprensión de las reacciones emocionales esperadas ante COVID-19, los factores individuales protectores y de riesgo, la patología psiquiátrica y las distintas intervenciones que pueden ponerse en práctica para apoyar las diversas reacciones presentadas.

\section{Factores protectores y de riesgo para la salud mental en personal de salud}

La enfermedad por SARS-Cov-2 (COVID-19) implica retos específicos para los trabajadores de la salud que predisponen a un mayor monto de estrés. Su alto contagio ha generado numerosos reportes de la enfermedad e, incluso, la muerte del personal en distintas partes del mundo, lo que genera un temor real en la atención a los pacientes, muchos de ellos sin diagnóstico al primer contacto. El manejo requiere ser especialistas de áreas críticas, rebasado en número, lo que está requiriendo la participación de médicos y enfermeras no especialistas o en formación de otras áreas.,
Las diversas manifestaciones clínicas, la falta de algoritmos claros para el manejo o deficiente difusión de los desarrollados, la elevada mortalidad y tórpida evolución de muchos de los casos, pueden generar una sensación de impotencia, incertidumbre y frustración entre los médicos tratantes. Otros factores inherentes a la atención de pacientes COVID-19 son la estigmatización social de la población, los estrictos requerimientos de bioseguridad, la incomodidad del equipo protector, la carencia de equipos suficientes, el aislamiento en áreas específicas para la atención de pacientes COVID-19, la necesidad de mantenerse en hipervigilancia constante para no contaminarse, la falta de espontaneidad en la convivencia con el resto de compañeros, la sensación de tener huecos de información con procedimientos en pacientes infectados, la sensación de injusticia en torno a la repartición de trabajo por servicios o niveles de puesto; el temor de contagiar a familia, pareja y amigos, que lleva a algunos médicos o enfermeras a estrategias de aislamiento y el cansancio físico.,

Al estrés de la atención de los pacientes infectados por el nuevo coronavirus SARS-CoV-2 se suman estresores laborales preexistentes, descritos en la bibliografía, entre ellos: la falta de correspondencia de salarios y la carga de trabajo, conflictos al interior de los servicios y otros aspectos organizacionales, que en la bibliografía se asocian con elevada prevalencia del "síndrome de burn-out" que constituye, per se, un factor de vulnerabilidad previa para manifestar síntomas físicos o emocionales y que se ha vinculado con un incremento en el riesgo de cometer errores en el desempeño hospitalario.

La remuneración de los profesionales de la salud en las instituciones requiere que un gran porcentaje de ellos labore más de un turno, lo que implica jornadas que rebasan, por mucho, las 48 horas semanales recomendadas. 
No todos los trabajadores de la salud experimentan el mismo impacto emocional, lo que implica que existen factores de riesgo, o protectores, para contender con el estrés psicológico de la pandemia.

Entre los elementos que deben tomarse en cuenta para evaluar la respuesta psíquica ante COVID-19 están los factores sociodemográficos, las demandas sociales y laborales, la estructura de personalidad, las estrategias de afrontamiento empleadas y las redes de apoyo social con que cuenta cada individuo.

Los mecanismos de afrontamiento son las estrategias cognitivas y las conductas que se ponen en marcha para enfrentar la ansiedad en la interacción con el ambiente. El afrontamiento poco adaptativo consiste en negar o evitar las emociones o situaciones que se enfrentan. Muchos médicos, predominantemente varones, están culturalmente troquelados para ignorar sus afectos, priorizando el trabajo sobre sus necesidades personales, evitando mostrar signos de temor o cansancio, lo que consideran "debilidad" $y$, por tanto, son quienes menos buscan apoyo emocional ante las crisis, y tienden a correr mayores riesgos, quitándose lentes o cubrebocas en áreas no COVID-19, por considerar que es emasculante. ,

El ser mujer, estar embarazada o tener hijos, han sido factores relacionados con mayor estrés. Está reportado que las mujeres tienen mayor temor al contagio, tanto personal como de su familia, en comparación con los hombres y son más vulnerables a padecer una sumatoria de factores de riesgo biológicos y sociales para tener alguna afección de salud mental.

El porcentaje de mujeres dentro del personal de salud a distintos niveles es mayoritariamente femenino y, pese a ello, la perpetuación de los roles y estereotipos de género impone de forma subliminal el cuidado de hijos y familiares enfermos y el trabajo de casa. Por eso, esta crisis coloca al personal femenino en una situación de sobrecarga emocional, poniendo en evidencia la inequidad en las labores de crianza y del hogar. Está documentado el incremento de violencia de género durante las pandemias.

Las familias monoparentales enfrentan el reto de acudir al centro hospitalario sin una red de apoyo para el cuidado de los hijos. Algunas familias, aparentemente funcionales, se confrontan con la realidad: infidelidades encubiertas y la patología de los distintos miembros de la familia (adicciones, trastornos alimentarios, hiperactividad, conductas disociales) se hacen evidentes, lo que se suma a la carga emocional de los trabajadores de la salud.

En muchas ocasiones, los problemas en el sueño se cubren con la automedicación de hipnóticos de alto potencial adictivo, contrarrestando la somnolencia diurna con estimulantes. Otros factores de riesgo son la historia sumatoria de exposición a otros eventos adversos en la vida, la coexistencia previa de psicopatología y las conductas adictivas.

También existen factores protectores que evitan la emergencia de problemas emocionales durante la pandemia. Entre ellos: el afrontamiento positivo, con actitudes resolutivas, la planeación de soluciones realistas a la problemática enfrentada, autoafirmación y la introspección, canalizan los sentimientos que emergen ante el estrés en actividades productivas y el sentido del humor.

Otros factores son: la valoración del autocuidado, la capacidad de pedir ayuda, la 
espiritualidad, llevar un estilo de vida saludable, tener pareja estable, contar con redes de apoyo, tener otras habilidades y pasatiempos: pintar, escuchar música, leer, escribir, ejercitarse, etc.

La resiliencia es un factor protector individual ante el estrés, es esa capacidad en la que se enlazan aspectos biológicos y en el desarrollo de la personalidad, para lidiar con la adversidad y recuperarse de forma espontánea, I@s sujet@s que poseen resiliencia, tienden a tener una personalidad bien estructurada, son tenaces y optimistas, mostrando menor riesgo de enfermedad mental.

\section{Reacciones emocionales ante la exposición a estrés}

Las primeras versiones médicas del estrés se apoyan en la idea de que el organismo es un sistema encargado de mantener el equilibrio interno, mediante mecanismos de retroalimentación planteados por Cannon, en 1932. Dicho modelo implica estabilidad, retroalimentación negativa e imposibilidad de transformación y, por tanto, crecimiento. Hans Seyle, en 1936, detalló el proceso biológico a través del cual se genera la cascada de estrés y supuso que la secuencia de respuestas decidía si el organismo se sobreponía a la adversidad cualquiera que fuese. Figura 1

Durante la respuesta al estrés se activan dos tipos diferentes de circuitos en el sistema nervioso central. Por una parte, se involucran respuestas del sistema nervioso autónomo, tanto simpáticas como parasimpáticas, mientras que en el sistema nervioso central se realiza una interpretación cortical del estresor, aunada a la respuesta del sistema límbico y del eje hipotálamo-hipófisisadrenal.

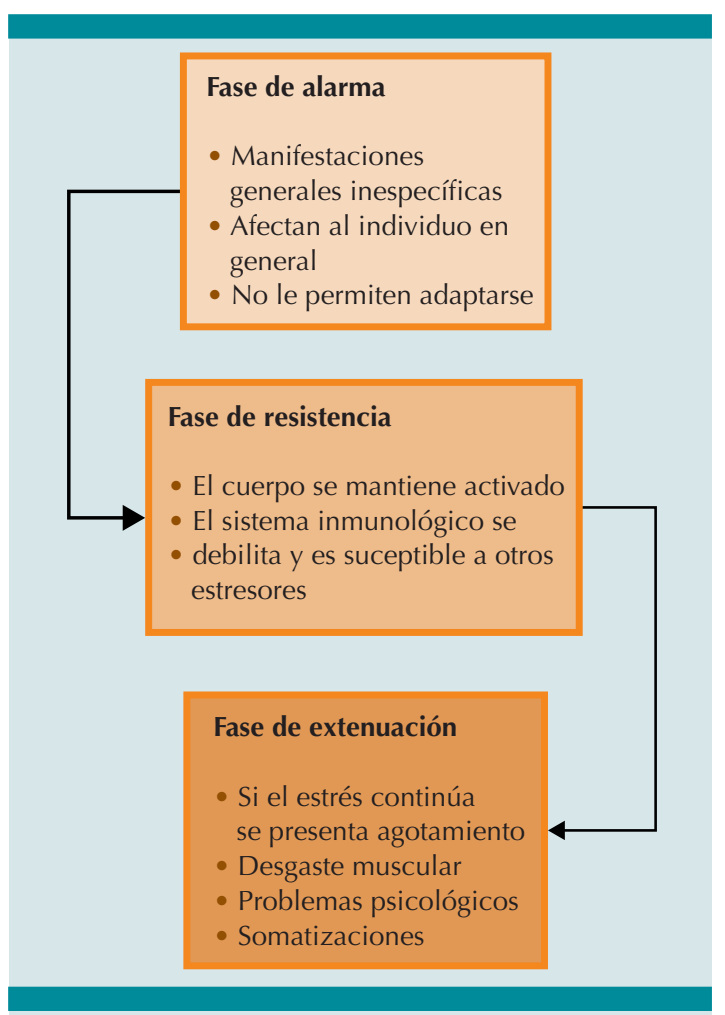

Figura 1. Respuestas biológicas ante estrés (H Selye).

El hipotálamo activa al sistema neuroendocrino, secreta hormonas como la hormona liberadora de corticotropina, que estimula la secreción de glucocorticoides y generan altas concentraciones de cortisol y, dependiendo del tiempo de exposición al estresor, puede generar disfunción de la respuesta inmunitaria.

Las reacciones emocionales pueden tener una intensidad leve o excesiva y pueden hacer que las personas logren desarrollar medidas de afrontamiento asertivas con el fin de adaptarse a ese nuevo entorno, en este caso a una pandemia causada por el virus SARS-COV-2 (COVID-19) $\mathrm{o}$, por el contrario, genera conductas desadaptativas a nivel emocional, conductual o cognitivo, que solo limitan el funcionamiento de quien las 
presenta y, en muchas ocasiones, no le permite una adecuada toma de decisiones.

En situaciones de normalidad, la corteza prefrontal regula las emociones, pensamientos y conducta; sin embargo, cuando el estrés ocasiona la pérdida de equilibrio, pueden aparecer fallas cognitivas, conductas disfuncionales y síntomas físicos pasajeros. De no remitir la exposición al estrés o ante la dificultad para adaptarse, pueden emerger cuadros psicopatológicos, como el trastorno de estrés postraumático, los trastornos depresivos, trastornos psicosomáticos o conductas suicidas. Figura 2

De acuerdo con el DSM-5 y el CIE-10 la reacción al estrés agudo es una respuesta transitoria que aparece ante un estresor psicológico excepcional, que generalmente remite en horas o días. Existe un

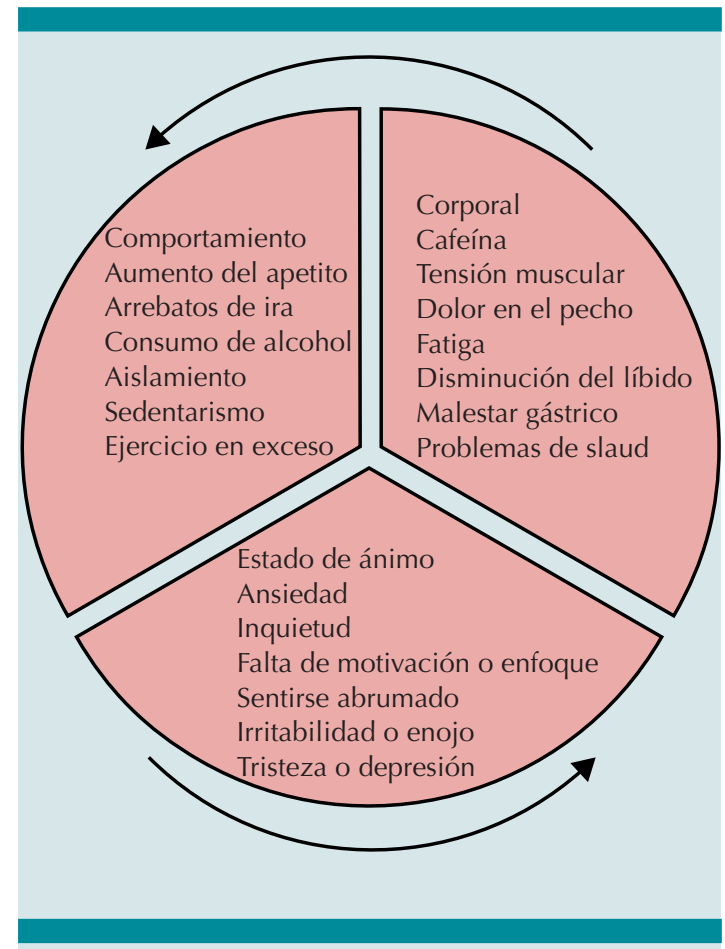

Figura 2. Manifestaciones conductuales, afectivas y somáticas ante el estrés. periodo inicial de "embotamiento" con ligera reducción del campo de la conciencia, disminución de la atención, incapacidad para asimilar estímulos y desorientación. Por lo general, también coexisten signos neurovegetativos: taquicardia, sensación de disnea, diaforesis y rubor.

Existen algunos indicadores que traducen que el estrés está generando conductas desadaptativas: agotamiento mental, pérdida progresiva del interés en actividades cotidianas, apatía, comportamientos que van de la indiferencia al distanciamiento $y$, en algunas ocasiones, desesperanza. Esos síntomas pueden generar disfunción laboral, familiar y social.

La exposición repetida o continua ante el estrés, como la observada en la pandemia por COVID-19, afecta las funciones cognitivas, afectivas y conductuales del sujeto y de no resolverse generan alteraciones metabólicas e inmunológicas que debilitan al organismo para hacer frente a procesos infecciosos, incrementando el riesgo del personal expuesto a enfermar.

Las reacciones emocionales reportadas como frecuentes por el personal de salud son: miedo, irritabilidad, enojo, labilidad emocional, tristeza, culpa, fallas de atención, concentración, alteraciones del sueño y del apetito.

Los trastornos adaptativos emergen cuando el sujeto enfrenta un estresor prolongado o mayor al esperado, por lo que las reacciones emocionales y conductuales se tornan persistentes y contribuyen a la aparición de síntomas de ansiedad o depresión, que generan una distorsión en la percepción de sí mismo y del mundo que lo rodea. Estos síntomas suelen aparecer durante los primeros tres meses desde el suceso estresante y desaparecen alrededor de seis meses después de finalizada la exposición. 
Los trastornos adaptativos se encuentran en el límite entre la normalidad y la patología mental, es decir, que son una especie de puente entre las personas que logran adaptarse a la nueva condición y las que se sienten sobrepasadas por lo que está aconteciendo y desarrollan trastornos psiquiátricos.

Entre los síntomas y signos de los trastornos de adaptación se encuentran:

- Sentimientos de tristeza, desesperanza o no disfrutar cosas placenteras.

- Llorar con frecuencia.

- Preocupación contínua, ansiedad y tensión mental.

- Trastornos del sueño.

- Falta de apetito.

- Dificultad para concentrarse.

- Sensación de estar sobrepasado.

- Tener dificultades para desenvolverse en las actividades diarias.

- Apartarse de las fuentes de apoyo social.

- Evitar asuntos importantes, como ir al trabajo o pagar cuentas.

- Deseos de morir o conductas autolesivas.

Trastornos psiquiátricos que pueden presentarse en el personal de salud

La emergencia de síntomas psiquiátricos en los trabajadores de la salud es multifactorial. Existe discrepancia en la bibliografía en torno a si los trastornos emergentes son aspectos adaptativos ante la pandemia, o exacerbaciones de trastornos preexistentes que, en situación de crisis, se manifiestan de forma exacerbada, cuando hay datos en la personalidad previa que favorecen un nivel inadecuado de adaptación.

El personal de salud, en muchas ocasiones, es renuente a buscar atención médico-psiquiátrica, ya sea por aspectos de personalidad, o por el estigma social que representa ser visualizados como "débiles" o "incapaces" de controlar sus propias emociones. Cuando la reacción por estrés agudo no se resuelve, puede favorecer un trastorno de estrés postraumático.

Otros trastornos que pueden manifestarse o exacerbarse en situaciones de crisis son los de ansiedad, depresivos, por abuso de sustancias: alcohol, tabaco, marihuana, estimulantes y ansiolíticos; también existen reportes de conductas alimentarias compulsivas, como el trastorno por atracones. La depresión, la ansiedad y el abuso de sustancias pueden paparecer de manera independiente o asociados con trastorno de estrés postraumático.

El trastorno de estrés postraumático, con una prevalencia de $9 \%$, incluye pensamientos intrusivos frecuentes (sueños, recuerdos vívidos, etc.), conductas evitativas (dejar de acudir al lugar donde sucedió el evento), alteraciones en la cognición y el estado de ánimo (problemas de memoria, distorsiones del pensamiento, afectos bajos o desinterés en lo cotidiano), y alteraciones del estado de alerta y de reacción (problemas para dormir, conductas impulsivas, irritabilidad). El diagnóstico es clínico y amerita la presencia de los grupos sintomáticos mencionados, y el antecedente del evento traumático. Se requiere una evaluación acuciosa porque las personas tienden a minimizar los síntomas en un intento de control narcisista o de rigidez de sus respuestas.

La depresión es una enfermedad de alta prevalencia en todo el mundo, frecuentemente subdiagnosticada y no tratada de manera adecuada. Existen reportes recientes que refieren la exacerbación de trastornos depresivos en el personal ante la pandemia de COVID-19 en los diferentes centros hospitalarios. El diagnóstico es clínico y debe incluir un estado de ánimo 
bajo, tristeza constante, ideas de desesperanza (la sensación de la inutilidad de lo que se haga), síntomas corporales como astenia y adinamia. Con frecuencia pueden aparecer ideas de muerte o ideación suicida, que constituyen el mayor riesgo de estos cuadros y que se han reportado en médicos, con patología previa, que han sucumbido ante la impotencia. ${ }^{25}$

Los trastornos de ansiedad se manifiestan, básicamente, por el temor ante la incertidumbre, la sensación de pérdida del control, y la coexistencia de síntomas psicofisiológicos concomitantes: taquicardia, diaforesis, disnea y que, en alguna de sus variedades, puede llevar a una crisis ansiosa o ataque de pánico, que paraliza al sujeto, le impide la toma de decisiones organizadas para su funcionamiento y limita la vida cotidiana tanto en su aparición, como el resto de la vida, de no recibir un tratamiento adecuado. ${ }^{25}$ Por último, puede aumentar el número de casos de abuso de sustancias, frecuente entre el personal médico, entre otras razones debido a los niveles de respuesta al estrés.

El consumo de alcohol, marihuana y benzodiacepinas es una forma de automedicación ante la ansiedad y al consumirlos fuera de control profesional, lleva al gremio médico a tener en mayor proporción tasas altas de consumo de estas sustancias que, de no ser tratado en fases tempranas, existe el riesgo de dependencia y otras complicaciones psiquiátricas.

\section{Atención de la problemática de salud mental en personal de salud}

La intervención inicial de apoyo busca brindar al personal de salud estrategias para enfrentar de la mejor manera la crisis por COVID-19.

\section{Intervención de primer orden}

Se proponen cinco componentes básicos: el contacto emocional, la focalización del problema, la evaluación de las soluciones posibles, el análisis de toma de decisiones y el seguimiento a corto plazo. Cuadro 1

Si la respuesta al estrés agudo no remite o al encontrarse psicopatología previa o comorbilidad psiquiátrica debe iniciarse un tratamiento farmacológico específico para las entidades nosológicas diagnosticadas. Cuadro 2

\section{Trastorno por estrés agudo}

La mayoría de los pacientes tendrá remisión de los síntomas sin necesidad de tratamiento.

Cuadro 1. Intervención en crisis

\begin{tabular}{|ll|}
\hline Hacer contacto emocional & $\begin{array}{l}\text { Hacer sentir al paciente o sobreviviente que es escuchado, comprendido, aceptado } \\
\text { y apoyado. Comunicar interés, simpatía, invitar a dialogar, resumir y reflejar hechos } \\
\text { y sentimientos. }\end{array}$ \\
\hline $\begin{array}{l}\text { Dimensionar el problema } \\
\text { Evaluar las soluciones posibles en el presente y futuro inmediatos a la crisis. }\end{array}$ & $\begin{array}{l}\text { El objetivo aquí es identificar una o más soluciones o necesidades inmediatas y } \\
\text { posteriores. Nueva conducta del paciente, redefinición del problema, ayuda externa } \\
\text { o cambio ambiental. }\end{array}$ \\
\hline $\begin{array}{l}\text { Ayudar a la toma de decisiones para } \\
\text { acciones concretas }\end{array}$ & $\begin{array}{l}\text { El objetivo es implementar soluciones inmediatas intentadas al encontrar necesidades } \\
\text { inmediatas. Se puede tomar una actitud facilitadora o una actitud directiva. }\end{array}$ \\
\hline Seguimiento & $\begin{array}{l}\text { El seguimiento concluye cuando el profesional puede asegurarse de que el paciente } \\
\text { hizo un enlace con sus necesidades. }\end{array}$
\end{tabular}


Cuadro 2. Tratamiento farmacológico de psicopatología emergente

\begin{tabular}{|c|c|c|c|}
\hline Familia de Medicamentos & Fármacos & Tipo de evidencia & Duración \\
\hline $\begin{array}{l}\text { Inhibidores de la recaptura } \\
\text { de serotonina (ISRS) }\end{array}$ & Sertralina, Paroxetina & $\begin{array}{l}\text { Estudios clínicos controlados, } \\
\text { aleatorios y multicéntricos han } \\
\text { demostrado buena eficacia }\end{array}$ & $\begin{array}{l}6 \text { a } 12 \text { meses TEPT } \\
\text { agudo } \\
>12 \text { meses TEP } \\
\text { crónico }\end{array}$ \\
\hline Inhibidores duales & Mirtazapina & $\begin{array}{l}\text { Estudios en refugiados, más del } \\
75 \% \text { reducción en pesadillas y } \\
\text { bloqueo de la memoria del sueño } \\
\text { al despertar }\end{array}$ & $\begin{array}{l}\text { Duración mayor } 6 \\
\text { meses }\end{array}$ \\
\hline Benzodiacepinas & $\begin{array}{l}\text { Clonazepam, alprazolam, } \\
\text { lorazepam }\end{array}$ & $\begin{array}{l}\text { Estudios controlados, mejoría en } \\
\text { insomnio y ansiedad, no son los } \\
\text { medicamentos de elección }\end{array}$ & $\begin{array}{l}\text { Duración breve, } \\
\text { menor a } 2 \text { meses }\end{array}$ \\
\hline Antipsicóticos & $\begin{array}{l}\text { Atipicos: olanzapina, quetiapina, } \\
\text { risperidona y aripiprazol }\end{array}$ & $\begin{array}{l}\text { Han demostrado utilidad para } \\
\text { disminuir agresividad y sintoma- } \\
\text { tología psicótica, en combinación } \\
\text { con ISRS }\end{array}$ & Duración breve \\
\hline $\begin{array}{l}\text { Moduladores } \\
\text { Del estado de ánimo }\end{array}$ & Topiramato, gabapentina & $\begin{array}{l}\text { Mejoría en la calidad de sueño, } \\
\text { disminución de pesadillas hasta } \\
\text { en el } 77 \%\end{array}$ & meses a 1 año \\
\hline
\end{tabular}

Algunos expertos recomiendan la recapitulación sistemática para ayudar a todos lo que estuvieron implicados en el evento traumático. Las intervenciones se basan en la elaboración del evento traumático, a través de la reintegración de los sucesos traumáticos, con el fin de que estos no sigan causándole dolor ni sufrimiento, ya sea en sesiones individuales o grupales. En estos casos ha demostrado eficacia la técnica de desensibilización y reprocesamiento de movimientos oculares.

\section{Trastorno de estrés postraumático}

Los inhibidores de la recaptura de serotonina son los medicamentos que han demostrado mayor efectividad en el control de los síntomas de pacientes con trastorno por estrés postraumático.

Los resultados de los ensayos clínicos con inhibidores de la recaptura de serotonina muestran mayor efectividad comparada con el placebo. Entre las estrategias psicológicas, la terapia cognitivo conductual ha demostrado eficacia en la reducción de los síntomas y prevención de la recurrencia, si se inicia en los primeros meses después del evento traumático.

\section{Trastorno de ansiedad (“crisis de pánico")}

El tratamiento de elección en una crisis de pánico es una benzodiacepina a dosis baja. La combinación de psicoterapia y benzodiacepinas vs monoterapia conductual solo es eficaz durante la primera fase. En la fase de mantenimiento no se recomiendan las benzodiacepinas y se sugieren los inhibidores de la recaptura de serotonina o imipramina, que disminuyen los síntomas de ansiedad anticipatoria, evitación agorafóbica y, por ende, la calidad de vida.

\section{Trastornos depresivos}

El tratamiento de los trastornos depresivos contempla la indicación de inhibidores de la recaptura de serotonina y la psicoterapia. La 
complicación más grave de la depresión es el suicidio. En conductas autolesivas, el tratamiento farmacológico contempla a los inhibidores de la recaptura de serotonina, ansiolíticos, antipsicóticos y, dependiendo de la letalidad y posibilidades de vigilancia, puede plantearse la hospitalización.

\section{Abuso de alcohol o sustancias}

El tratamiento de los trastornos por abuso de sustancias incluye el retiro gradual de la sustancia y la prescripción de medicamentos para disminuir la ansiedad ante el consumo y evitar los efectos físicos de la abstinencia. La asistencia a grupos de apoyo ha demostrado un beneficio sinérgico.

\section{CONCLUSIONES}

La pandemia por COVID-19 es un factor de riesgo de problemas de salud mental en los trabajadores de la salud que requiere evaluación y atención oportuna por parte de los especialistas en salud mental.

\section{REFERENCIAS}

1. https://www.who.int/topics/mental_health/es/ (consultado en línea 2020, mayo 9)

2. Holmes EA, et al. Multidisciplinary research priorities for the COVID-19 pandemic: a call for action for mental health science. doi:10.1016/S2215-0366(20)30168-1.

3. Ran L, et al. Risk factors of healthcare workers with Coronavirus disease 2019: A retrospective cohort study in a designated Hospital of Wuhan in China. Clin Infect Dis. 2020; 287. https://doi.org/10.1093/cid/ciaa287

4. Elkholy AA, et al. MERS-CoV infection among healthcare workers and risk factors for death: Retrospective analysis of all laboratory-confirmed cases reported to WHO from 2012 to 2 June 2018. J Infect Public Health. 2020;13(3):418-422. doi:10.1016/j.jiph.2019.04.011.

5. Briefing note on addressing mental health and psychosocial aspects of COVID-19. 1.0 Inter-Agency Standing Committee IASC Reference group for MHPSS-COVID19-Briefing-NoteFINAL_26-February-2020-UN. https://MHPSS-COVID19Briefing-Note-FINAL_26-February-2020-UN.)
6. https://www.who.int/publications-detail/coronavirusdisease-(covid-19)-outbreak-rights-roles-and-responsibilities-of-health-workers-including-key-considerations-foroccupational-safety-and-health

7. Miranda V, et al. Prevalencia del síndrome de burnout en personal de enfermería de dos instituciones de salud. Rev Enferm Inst Mex Seguro Soc. 2016; 24 (2): 115-22.

8. Terrones JF, et al. Síndrome de burnout en médicos residentes del Hospital General de Durango, México. Rev Med Inst Mex Seguro Soc. 2016; 54 (2): 242-8.

9. Berdahk J, et al. How masculinity contests undermine organizations and what to do about it. https://hbr. org/2018/11/:

10. Glick P. Masks and emasculation. Why some men refuse to take safety precautions.https://blogs.scientificamerican. com/observations/masks-and-emasculation-why-somemen-refuse-to-take-safety-precautions/.

11. Panchal N, Kamal R. The Implications of COVID-19 for Mental Health and substance abuse. https://www.kff. org/health-reform/issue-brief/the-implications-of-covid19-for-mental-health-and-substance-use/.)

12. Wenham C, et al. COVID-19: the gendered impacts of the outbreak. Lancet; 2020 (395): 846-48. https://doi. org/10.1016/S0140-6736(20)30526-2

13. Díaz-Campo Y, et al. La calidad del sueño en los profesionales sanitarios de dos Áreas de Salud de Castilla-La Mancha. Rev Clin Med Fam. 2008; 2 (4): 2386-820.

14. Izquierdo-Sotorrío E. Los mecanismos de defensa desde la perspectiva de género y su impacto sobre la salud. Revista Digital de Medicina Psicosomática y Psicoterapia. www. researchgate.net/publication/277923543

15. Sehmi $\mathrm{R}$, et al. No man is an island: social resources, stress and mental health at mid-life. Br J Psychiatry. 2019; 4 (1):17. https://doi.org/10.1192/bjp.2019.25

16. Cai $\mathbf{W}$, et al. A cross-sectional study on mental health among health care workers during the outbreak of Corona Virus Disease 2019. Asian J Psychiatr. 2020; 51(5):102-11. 10.1016/j.ajp.2020.102111

17. Lazarus, R.S. (cop. 2000) Estrés y emoción. Manejo e implicaciones en nuestra salud. Bilbao. Desclée de Brouwer

18. Rodríguez-Fernández JM, et al. Neurobiology of Acute and Chronic Stress: Its Effect on the Hypothalamo-PituitaryAdrenal Axis and Memory Universitas Médica. 2013;54(7): 472-94. http://revistas.javeriana.edu.co/index.php/vnimedica/article/view/16266

19. www.mayoclinic.org/es-es/healthy-lifestyle/stressmanagement/basics/stress-basics/hlv-20049495 (consultada en linea2020, mayo 5)

20. Fernández-Abascal E. G y Palmero F (1999). Emociones y salud. Barcelona: Ariel

21. Reissman D, et al. Pandemic influenza preparedness: adaptive responses to an envolving challenge. J Homel Secur Emerg Manag. 2006; 3(2). https://doi.org/10.2202/15477355.1233 
22. Strain JJ, et al. Trastornos adaptativos. En: Gelder MG, Lopez-Ibor JJ, Andreasen N. Tratado de Psiquiatría. Tomo II. Barcelona: Ars Medica 2003, 922-33

23. Instituto Nacional de Perinatología "Isidro Espinosa de los Reyes" lineamiento técnico. Abordaje de infección por covid-19 en el periodo perinatal en contingencia abril 2020. http:// inper.mx/descargas-2019/pdf/LineamientoINPerCOVID19_2.

24. National Institute of Mental health. USA. Post-traumatic stress disorder. http://www.fda.gov/.

25. American Psychiatric Association. Diagnostic and Statistical Manual of Mental Disorders (DSM-5). 5th ed. Washington: American Psychiatric Association, 2013.

26. Kumar P, Basu D. Substance Abuse by Medical Students and Doctors J Indian Med Assoc. 2000 Aug; 98 (8): 447-52.
27. Martínez-Lanz, et al. Consumo de alcohol y drogas en personal de salud: algunos factores relacionados. Salud Ment. 2004; 27 (6): 17-27.

28. Wampold BE, et al. In pursuit of truth: A critical examination of meta-analyses of cognitive behavior therapy. Psychother Res. 2017; 27 (1): 14-32. https://doi.org/10 $.1080 / 10503307.2016 .1249433$

29. Zugliani MM, et al. Pharmacological and neuromodulatory treatments for panic disorder: Clinical Trials from 2010 to 2018. Psychiatry Investig. 2019;16(1):50-58. 10.30773/pi.2018.12.21.1

30. Gautam S, et al. Clinical Practice Guidelines for the management of Depression. Indian J Soc Psychiatry. 2017; 59 (Suppl 1): 34-50. doi: 10.4103/0019-5545.196973. 